\title{
Examples of nonclassical feedback control problems
}

\author{
Alberto Bressan and Deling Wei
}

\begin{abstract}
We consider a control system with "nonclassical" dynamics: $\dot{x}=f\left(t, x, u, D_{x} u\right)$, where the right hand side depends also on the first order partial derivatives of the feedback control function. Given a probability distribution on the initial data, we seek a feedback $u=u(t, x)$ which minimizes the expected value of a cost functional. Various relaxed formulations of this problem are introduced. In particular, three specific examples are studied, showing the equivalence or non-equivalence of these approximations.
\end{abstract}

Mathematics Subject Classification. 49N25, 49N35.

\section{Introduction}

Consider a controlled system whose state $x \in \mathbb{R}^{n}$ evolves according to

$$
\dot{x}=f(t, x, u, D u) \text {. }
$$

Here $u=u(t, x)$ is a feedback control, taking values in a set $U \subseteq \mathbb{R}^{m}$, while the upper dot denotes a derivative w.r.t. time. The dependence of the right hand side of (1.1) on the Jacobian matrix $D u=\left(\partial u_{i} / \partial x_{j}\right)$ makes the problem "nonclassical". Control systems with this generalized dynamics arise naturally in connection with closed-loop Stackelberg solutions to differential games $[5,6,8]$.

As remarked in [8], it is useful to compare (1.1) with the relaxed system

$$
\dot{x}=f(t, x, u, v),
$$

where $u \in \mathbb{R}^{m}, v \in \mathbb{R}^{m \times n}$ are now regarded as independent controls. Clearly, every solution of (1.1) yields a solution of (1.2), simply by choosing $v=D u$. On the other hand, given an initial data

$$
x(0)=x_{0},
$$

Dedicated to Arrigo Cellina: teacher, mentor and friend. 
let $t \mapsto x^{*}(t)$ be the solution of the Cauchy problem (1.2)-(1.3) corresponding to the open-loop measurable controls $u(t), v(t)$. If we choose

$$
u^{*}(t, x)=u(t)+v(t) \cdot\left(x-x^{*}(t)\right)
$$

for all $x$ in a neighborhood of $x^{*}(t)$, then $x^{*}$ satisfies also the original equation (1.1).

Given a cost functional such as

$$
J \doteq \int_{0}^{T} L(t, x(t), u(t, x(t))) d t
$$

for a fixed initial condition (1.3) the infimum of the cost over all admissible controls is thus the same for trajectories of (1.1) or (1.2). The main difficulty in the study of this minimization problem lies in the fact that the control $v$ can be arbitrarily large and comes at zero cost. Therefore, optimal trajectories may well have impulsive character. For optimization problems with impulsive controls we refer to $[7,10,11]$.

Aim of the present paper is to understand what happens in the case where the initial data is not assigned in advance, and one seeks a feedback $u=u(t, x)$ that is optimal in connection with a whole collection of possible initial data. Motivated by problems related to differential games $[5,6,8]$, we consider a system whose state is described by a pair of scalar variables $(x, \xi) \in \mathbb{R} \times \mathbb{R}$. For simplicity, we also assume that the control variable $u(t) \in \mathbb{R}$ is one-dimensional. Let the system evolve in time according to the ODEs

$$
\left\{\begin{array}{l}
\dot{x}=f(t, x, \xi, u) \\
\dot{\xi}=g\left(t, x, \xi, u, u_{x}\right)
\end{array}\right.
$$

where $f, g$ are Lipschitz continuous functions of their arguments. We assume that the initial data for the variable $x$ is distributed according to a probability distribution $\mu$ on $\mathbb{R}$, while $\xi$ is determined by a constraint of the form

$$
\xi(0)=h(x(0)) \text {. }
$$

Here $h: \mathbb{R} \mapsto \mathbb{R}$ is some continuous function. A feedback control $u=u(t, x)$ is sought, in order to minimize the cost functional

$$
J(u, \mu) \doteq E^{\mu}\left[\int_{0}^{T} L(t, x(t), \xi(t), u(t, x(t))) d t\right] .
$$

Here $E^{\mu}$ denotes the conditional expectation w.r.t. the probability distribution $\mu$ on the set of initial data.

In general, a optimal feedback may not exist, within the class of $\mathcal{C}^{2}$ functions. Indeed, it is quite possible that the optimal control will be discontinuous w.r.t. the space variable $x$, or even measure-valued. To bypass all difficulties stemming from the possible lack of regularity, we consider the family $\mathcal{U}$ of all $\mathcal{C}^{2}$ functions $u:[0, T] \times \mathbb{R} \mapsto \mathbb{R}$. For each feedback control $u \in \mathcal{U}$ the equation (1.6) uniquely determines a flow on $\mathbb{R}^{2}$. We denote by

$$
t \mapsto\left(\begin{array}{l}
x(t) \\
\xi(t)
\end{array}\right)=\Psi_{t}^{u}\left(\begin{array}{l}
\bar{x} \\
\bar{\xi}
\end{array}\right)
$$


the solution of the Cauchy problem

$$
\frac{d}{d t}\left(\begin{array}{l}
x(t) \\
\xi(t)
\end{array}\right)=\left(\begin{array}{c}
f(t, x(t), \xi(t), u(t, x(t))) \\
g\left(t, x(t), \xi(t), u(t, x(t)), u_{x}(t, x(t))\right)
\end{array}\right),
$$

with initial data

$$
\left(\begin{array}{l}
x(0) \\
\xi(0)
\end{array}\right)=\left(\begin{array}{l}
\bar{x} \\
\bar{\xi}
\end{array}\right)=\left(\begin{array}{c}
\bar{x} \\
h(\bar{x})
\end{array}\right) .
$$

Here $\bar{x} \in \mathbb{R}$ is a random variable, distributed according to the probability measure $\mu$. Let $\mu^{(t)}$ be the corresponding probability distribution at time $t$, defined as the push-forward of $\mu$ through the flow $\Psi_{t}^{u}$. This means

$$
\mu^{(t)}\left(\Psi_{t}^{u}(A)\right)=\mu(A)
$$

for every Borel set $A \subset \mathbb{R}^{2}$. The cost functional in (1.8) can be equivalently written as

$$
J(u, \mu)=\int_{0}^{T} E^{\mu^{(t)}} L(t, x, \xi, u(t, x)) d t,
$$

where $E^{\mu^{(t)}}$ denotes expectation w.r.t. the probability distribution $\mu^{(t)}$. We then consider

Problem 1. Determine

$$
J(\mu) \doteq \inf _{u \in \mathcal{U}} J(u, \mu)
$$

Describe a sequence of feedback controls $\left(u_{n}\right)_{n \geq 1}$ achieving the infimum in (1.12).

As it will be shown by some examples, the infimum in (1.12) may not be stable w.r.t. perturbations of the probability distribution $\mu$. A related question is to determine the value

$$
J^{w}(\mu) \doteq \liminf _{d(\tilde{\mu}, \mu) \rightarrow 0} \inf _{u \in \mathcal{U}} J(u, \tilde{\mu})
$$

where

$$
d(\tilde{\mu}, \mu)=\sup \left\{\left|\int \varphi d \tilde{\mu}-\int \varphi d \mu\right| ; \quad \varphi \in \mathcal{C}^{1},|\nabla \varphi| \leq 1\right\}
$$

is the Kantorovich-Wasserstein distance between two probability measures. One can think of $J^{w}$ as the lower semicontinuous regularization of $J$ w.r.t. the topology of weak convergence of measures.

In the case where $\mu$ is absolutely continuous with density $\phi$ w.r.t. Lebesgue measure, it is also meaningful to consider

$$
J^{s}(\mu) \doteq \liminf _{\|\tilde{\phi}-\phi\|_{\mathbf{L}^{1} \rightarrow 0}} \inf _{u \in \mathcal{U}} J(u, \tilde{\mu}),
$$

where $\tilde{\mu}$ is a probability measure having density $\tilde{\phi}$. In other words, $J^{s}$ is the lower semicontinuous regularization of $J$ w.r.t. a strong topology, corresponding to $\mathbf{L}^{1}$ convergence of the densities. 
As it will be shown by specific examples, the three values in (1.12)-(1.14) may well be different. In addition, by replacing $u_{x}$ with an independent control function $v$, from (1.9) one obtains the relaxed system

$$
\frac{d}{d t}\left(\begin{array}{l}
x(t) \\
\xi(t)
\end{array}\right)=\left(\begin{array}{c}
f(t, x(t), \xi(t), u(t, x(t))) \\
g(t, x(t), \xi(t), u(t, x(t)), v(t, x(t)))
\end{array}\right) .
$$

We shall denote by $\mathcal{J}(\mu, u, v)$ be the corresponding cost (1.8), with dynamics given at (1.15).

Remark 1. In general, the optimal control $u=u(t, x)$ which minimizes the expected cost (1.8) subject to the dynamics (1.9) will strongly depend on the probability distribution $\mu$ on the initial data. On the other hand, since the dynamics (1.15) does not involve derivatives of the control functions $u, v$, the optimal value can be achieved pointwise for each initial data $x(0), \xi(0)$. In this case, the same pair of feedback controls $\left(u^{*}, v^{*}\right)$ can be optimal for every probability distribution $\mu$ on the initial data.

We now introduce the set $\mathcal{V}$ of all $\mathcal{C}^{2}$ functions $v:[0, T] \times \mathbb{R} \mapsto \mathbb{R}$, and consider

Problem 2. Determine the optimal value for the relaxed problem

$$
J^{\text {relax }}(\mu) \doteq \inf _{(u, v) \in \mathcal{U} \times \mathcal{V}} \mathcal{J}(u, v, \mu)
$$

Describe a sequence of feedback controls $\left(u_{n}, v_{n}\right)_{n \geq 1}$ achieving the infimum in (1.16).

From the definitions, it is immediately clear that

$$
J^{\operatorname{relax}}(\mu) \leq J(\mu), \quad J^{w}(\mu) \leq J^{s}(\mu) \leq J(\mu)
$$

In this paper we analyze three specific examples. showing the differences between the original and relaxed formulations, and the possible obstructions encountered in the approximation of solutions (1.15) with solutions of (1.9). Motivated by these examples, general results on the equivalence between the various values in (1.17) will be proved in the forthcoming paper [2].

The underlying motivation for the present analysis comes from the theory of Stackelberg solutions in closed-loop form, for differential games. In one space dimension, this leads to a problem of the form (1.6), where $x$ is the state of the system, $u=u(t, x)$ is the feedback control implemented by the leader, and $\xi$ is the adjoint variable in the optimality equation determining the strategy of the follower. For a differential game, it is natural to put a probability distribution on the state $x$ at the initial time $t=0$, and a constraint of the type

$$
\xi(T)=h(x(T))
$$

on the adjoint variable at the terminal time $T$. The present research, dealing with the Cauchy problem where all data are given at time $t=0$, is intended to be an intermediate step toward the understanding of this boundary value problem, more relevant for game-theoretic applications. 


\section{A case of shrinking funnels}

Example 1. Consider the optimization problem

$$
\text { minimize: } J(u)=E^{\mu}\left[\int_{0}^{T}\left[x^{2}(t)+\xi^{2}(t)+u^{2}(t)\right] d t\right] .
$$

for the system with dynamics

$$
\left\{\begin{array}{l}
\dot{x}=u, \\
\dot{\xi}=\xi u_{x} .
\end{array}\right.
$$

Here $u=u(t, x)$ can range over the entire real line $\mathbb{R}$. As initial condition, assume that $\xi(0) \equiv 1$ while $x(0)$ is uniformly distributed on the interval $[0,1]$. Of course, this means that $\mu$ is the measure with density $\phi=\chi_{[0,1]}$ (the characteristic function of the unit interval) w.r.t. Lebesgue measure.

In this case, the corresponding relaxed problem, with $u_{x}$ replaced by an independent control function $v$, is decoupled. Indeed, it yields two independent problems:

minimize: $\quad J_{1}(u)=\int_{0}^{T}\left[x^{2}(t)+u^{2}(t)\right] d t, \quad$ with dynamics $\quad \dot{x}=u$,

$$
\text { minimize: } \quad J_{2}(v)=\int_{0}^{T} \xi^{2}(t) d t, \quad \text { with dynamics } \quad \dot{\xi}=\xi v .
$$

The first is a standard linear-quadratic optimal control problem. The optimal feedback is linear w.r.t. $x$, namely (Fig. 1, left)

$$
u^{*}(t, x)=\frac{e^{t-T}-e^{T-t}}{e^{t-T}+e^{T-t}} \cdot x .
$$

The second problem is solved by an unbounded impulsive control $v$ that instantly steers the component $\xi$ to the origin.

Returning to the original problem $(2.1)-(2.2)$, call $\phi(t, \cdot)$ the density of the probability distribution $\mu^{(t)}$. This function satisfies

$$
\phi_{t}+u \phi_{x}=-\phi u_{x}
$$

Calling $\xi(t, x)$ the value of $\xi(t)$ along a characteristic, i.e. at the point $(t, x(t))$, the second equation in (2.2) yields

$$
\xi_{t}+u \xi_{x}=\xi u_{x}
$$

Together, these two equations yield

$$
(\phi \xi)_{t}+u(\phi \xi)_{x}=0 .
$$

In the following, for $y \in[0,1]$ we shall denote by $t \mapsto x(t, y)$ the particular solution of the Cauchy problem

$$
\dot{x}=u(t, x), \quad x(0)=y .
$$



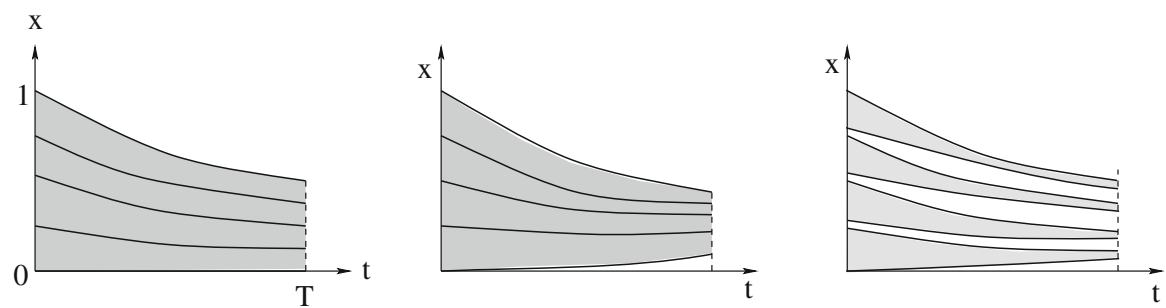

FiguRE 1. Left the optimal trajectories for the standard linear-quadratic optimization problem with dynamics (2.9) and cost (2.3) independent of $\xi$. Center the presence of a cost depending on $\xi$ renders more profitable a control where $u_{x}$ is large and negative. Hence the optimal solution, obtained by solving (2.16), should be supported on a smaller interval. Right if we allow small gaps in the support of the probability distribution $\mu$ on the initial data, then the minimum cost becomes arbitrarily close to the minimum cost for relaxed problem (2.3)-(2.4)

Expressing the feedback control in terms of this new variable: $u(t, y) \doteq u(t$, $x(t, y))$, we obtain

$$
u_{x}(t, x(t, y))=u_{y}(t, y) \cdot \phi(t, y)=\frac{u_{y}(t, y)}{\xi(t, y)} .
$$

The problem can thus be reformulated as

$$
\text { minimize: } \quad \int_{0}^{T} \int_{0}^{1}\left[x^{2}(t, y)+\xi^{2}(t, y)+u^{2}(t, y)\right] d y d t
$$

subject to

$$
\left\{\begin{array} { l } 
{ \dot { x } = u , } \\
{ \dot { \xi } = u _ { y } , }
\end{array} \quad \left\{\begin{array}{l}
x(0, y)=y \\
\xi(0, y)=1
\end{array}\right.\right.
$$

Since the evolution equation does not depend explicitly on $x, \xi$, the adjoint equations are

$$
\left\{\begin{array} { l } 
{ \dot { \lambda } _ { 1 } = - \partial L / \partial x = - 2 x , } \\
{ \dot { \lambda } _ { 2 } = - \partial L / \partial \xi = - 2 \xi , }
\end{array} \quad \left\{\begin{array}{l}
\lambda_{1}(T, y)=0 \\
\lambda_{2}(T, y)=0
\end{array}\right.\right.
$$

Hence

$$
\lambda_{1}(t, y)=\int_{t}^{T} 2 x(\tau, y) d \tau, \quad \lambda_{2}(t, y)=\int_{t}^{T} 2 \xi(\tau, y) d \tau .
$$

The maximality condition yields (Fig. 1, center)

$$
u(t, \cdot)=\underset{\omega(\cdot)}{\operatorname{argmin}} \int_{0}^{1}\left[\lambda_{1}(t, y) \omega(y)+\lambda_{2}(t, y) \omega_{y}(y)+\omega^{2}(y)\right] d y .
$$


Assume that, for a fixed time $t$, the function $u=u(t, y)$ provides the minimum in (2.12). Then, for every smooth function $\varphi:[0,1] \mapsto \mathbb{R}$, setting $u^{(\varepsilon)}(y)=u(t, y)+\varepsilon \varphi(y)$ one should have

$$
\begin{aligned}
0 & =\left.\frac{d}{d \varepsilon} \int_{0}^{1}\left[\lambda_{1}(t, y) u^{(\varepsilon)}(y)+\lambda_{2}(t, y) u_{y}^{(\varepsilon)}(y)+\left(u^{(\varepsilon)}\right)^{2}(y)\right] d y\right|_{\varepsilon=0} \\
& =\int_{0}^{1}\left[\lambda_{1}(t, y) \varphi(y)+\lambda_{2}(t, y) \varphi_{y}(y)+2 u(t, y) \varphi(y)\right] d y \\
& =\int_{0}^{1}\left[\lambda_{1}(t, y)-\lambda_{2, y}(t, y)+2 u(t, y)\right] \varphi(y) d y+\lambda_{2}(t, 1) \varphi(1)-\lambda_{2}(t, 0) \varphi(0) .
\end{aligned}
$$

Since the function $\varphi$ can be arbitrary, this yields the Euler-Lagrange equations

$$
u(t, y)=\frac{-\lambda_{1}(t, y)+\lambda_{2, y}(t, y)}{2} .
$$

together with the boundary conditions

$$
\lambda_{2}(t, 0)=\lambda_{2}(t, 1)=0 .
$$

Differentiating (2.13) w.r.t. $t$ and using (2.10), we obtain

$$
u_{t}(t, y)=\frac{\lambda_{2, t y}(t, y)-\lambda_{1, t}(t, y)}{2}=x-\xi_{y} .
$$

Using the identities

$$
x_{t t}(t, y)=u_{t}(t, y), \quad x_{y}(t, y)=\frac{1}{\phi(t, y)}=\xi(t, y),
$$

we eventually obtain the PDE

$$
x_{t t}+x_{y y}-x=0 .
$$

This is a linear elliptic equation, to be solved on the rectangle $[0, T] \times[0,1]$. From (2.9) and the terminal conditions in (2.10), using (2.13) one obtains the boundary conditions

$$
x(0, y)=y, \quad x_{t}(T, y)=u(T, y)=0 .
$$

Moreover,

$$
\left\{\begin{array}{l}
x_{y}(t, 0)=\xi(t, 0)=-\frac{1}{2} \lambda_{2, t}(t, 0)=0 \\
x_{y}(t, 1)=\xi(t, 1)=-\frac{1}{2} \lambda_{2, t}(t, 1)=0
\end{array}\right.
$$

because of (2.11) and (2.14).

By standard PDE theory, the linear elliptic boundary-value problem (2.16), (2.17), (2.18) has a unique solution. Particular solutions of (2.16) satisfying (2.18) can be obtained by separation of variables. For every integer $k \geq 0$ and coefficients $A_{k}, B_{k}$, one has the solution $x(t, y)=X_{k}(t) Z_{k}(y)$ where

$$
X_{k}(t)=A_{k} e^{\sqrt{1+k^{2} \pi^{2}} t}+B_{k} e^{-\sqrt{1+k^{2} \pi^{2}} t}, \quad Z_{k}(y)=\cos k \pi y,
$$


Imposing the additional boundary conditions (2.17) one obtains a representation of the solution as a Fourier series:

$$
\left\{\begin{aligned}
x(t, y)= & \frac{e^{t-2 T}+e^{-t}}{2\left(e^{-2 T}+1\right)}+\sum_{k=1}^{\infty}\left(\frac{e^{\sqrt{1+k^{2} \pi^{2}}(t-2 T)}+e^{-\sqrt{1+k^{2} \pi^{2}} t}}{e^{-2 \sqrt{1+k^{2} \pi^{2}} T}+1}\right) \\
& \times \frac{2\left((-1)^{k}-1\right)}{k^{2} \pi^{2}} \cos k \pi y, \\
\xi(t, y)= & x_{y}(t, x)=\sum_{k=1}^{\infty}\left(\frac{e^{\sqrt{1+k^{2} \pi^{2}}(t-2 T)}+e^{-\sqrt{1+k^{2} \pi^{2}} t}}{e^{-2 \sqrt{1+k^{2} \pi^{2}} T}+1}\right) \\
& \times \frac{2\left(1-(-1)^{k}\right)}{k \pi} \sin k \pi y .
\end{aligned}\right.
$$

Estimates on this solution can be obtained by comparison with upper and lower solutions [9]. Differentiating (2.16) w.r.t. $y$ one obtains a boundary value problem for $x_{y}$, namely

$$
\left(x_{y}\right)_{t t}+\left(x_{y}\right)_{y y}-x_{y}=0 \quad(t, y) \in[0, T] \times[0,1],
$$

with the boundary conditions

$$
\left\{\begin{array} { l } 
{ x _ { y } ( 0 , y ) = 1 , } \\
{ ( x _ { y } ) _ { t } ( T , y ) = 0 , }
\end{array} \quad \left\{\begin{array}{l}
x_{y}(t, 0)=0 \\
x_{y}(t, 1)=0
\end{array}\right.\right.
$$

A standard comparison argument here yields the lower bound

$$
x_{y}(t, y) \geq 0 \quad \text { for all } t, y \text {. }
$$

One can also consider the above problem on the half line, for $t \in[0,+\infty[$. In this case, (2.20) reduces to

$$
x(t, y)=\frac{e^{-t}}{2}+\sum_{k=1}^{\infty} e^{-\sqrt{1+k^{2} \pi^{2}} t} \cdot \frac{2\left((-1)^{k}-1\right)}{k^{2} \pi^{2}} \cos k \pi y .
$$

As $t \rightarrow \infty$, this solution approaches zero. Indeed,

$$
\|x(t, \cdot)\|_{\mathbf{L}^{\infty}([0,1])} \rightarrow 0, \quad\|\xi(t, \cdot)\|_{\mathbf{L}^{\infty}([0,1])} \rightarrow 0, \quad\|u(t, \cdot)\|_{\mathbf{L}^{\infty}([0,1])} \rightarrow 0 .
$$

We now study what happens if we allow arbitrarily small gaps in the support of the initial probability distribution $\mu$. For any $n \geq 2$, let $\mu_{n}$ be the probability distribution with density

$$
\phi_{n}(x)= \begin{cases}\frac{n}{n-1} & \text { if } x \in\left[a_{i}, b_{i}\right] \doteq\left[\frac{i-1}{n}, \frac{i}{n}-\frac{1}{n^{2}}\right] \\ & \text { for some } i=1, \ldots, n \\ 0 & \text { otherwise. }\end{cases}
$$

Clearly, $\lim _{n \rightarrow \infty}\left\|\phi_{n}-\phi\right\|_{\mathbf{L}^{1}}=0$. We claim that, as $n \rightarrow \infty$, the costs of the corresponding optimal solutions approach the minimum cost for (2.3). 
To prove this, consider any of the above intervals $\left[a_{i}, b_{i}\right] \subset[0,1]$. Let $x_{i}(t, y)$ be the solution of the linear elliptic boundary value problem

$$
x_{t t}+x_{y y}-x=0 \quad(t, y) \in[0, T] \times\left[a_{i}, b_{i}\right],
$$

with boundary conditions

$$
\left\{\begin{array} { l } 
{ x ( 0 , y ) = y , } \\
{ x _ { t } ( T , y ) = 0 , }
\end{array} \quad \left\{\begin{array}{l}
x_{y}\left(t, a_{i}\right)=0 \\
x_{y}\left(t, b_{i}\right)=0
\end{array}\right.\right.
$$

The solution to this boundary value problem can again be expressed as a Fourier series:

$$
\begin{aligned}
x_{i}(t, y)= & \left(\frac{e^{t-2 T}+e^{-t}}{e^{-2 T}+1}\right) \frac{b_{i}+a_{i}}{2}+\sum_{k=1}^{\infty}\left(\frac{e^{\lambda_{k}(t-2 T)}+e^{-\lambda_{k} t}}{e^{-2 \lambda_{k} T}+1}\right) \\
& \times \frac{2\left((-1)^{k}-1\right)\left(b_{i}-a_{i}\right)}{k^{2} \pi^{2}} \cos \frac{k \pi\left(y-a_{i}\right)}{b_{i}-a_{i}},
\end{aligned}
$$

where

$$
\lambda_{k}=\sqrt{1+\frac{k^{2} \pi^{2}}{\left(b_{i}-a_{i}\right)^{2}}} \geq 1 .
$$

In connection with the intervals $\left[a_{i}, b_{i}\right]$ defined at $(2.25)$, for $i=1, \ldots, n$ consider the funnels (Fig. 1, right)

$$
\Gamma_{i} \doteq\left\{(t, x) ; \quad t \in[0, T], \quad x=x_{i}(t, y) \quad \text { for some } y \in\left[a_{i}, b_{i}\right]\right\} .
$$

We claim that these funnels are pairwise disjoint. Indeed, consider the function

$$
z(t, y)=\frac{e^{t-2 T}+e^{-t}}{e^{-2 T}+1} y
$$

where $t \mapsto z(t, y)$ is determined as the unique solution to the two-point boundary value problem

$$
\ddot{z}(t)-z(t)=0 \quad \text { for } t \in[0, T], \quad z(0)=y, \quad \dot{z}(T)=0 .
$$

Then $z_{y}$ provides a solution to the elliptic boundary value problem

$$
w_{t t}+w_{y y}-w=0 \quad(t, y) \in[0, T] \times\left[a_{i}, b_{i}\right],
$$

with boundary conditions

$$
\left\{\begin{array}{l}
w(0, y)=1, \\
w_{t}(T, y)=0,
\end{array} \quad w\left(t, a_{i}\right)=w\left(t, b_{i}\right)=\frac{e^{t-2 T}+e^{-t}}{e^{-2 T}+1} .\right.
$$

On the other hand, the partial derivative $x_{i, y}$ provides a solution to the same equation (2.32), but with boundary conditions

$$
\left\{\begin{array}{l}
w(0, y)=1, \\
w_{t}(T, y)=0,
\end{array} \quad w\left(t, a_{i}\right)=\left(t, b_{i}\right)=0 .\right.
$$

By comparison, we obtain

$$
x_{y}(t, y) \leq z_{y}(t, y) \quad \text { for all }(t, y) \in[0, T] \times\left[a_{i}, b_{i}\right]
$$


When $y=\left(a_{i}+b_{i}\right) / 2$, from (2.28) it follows $x_{i}(t, y)=z(t, y)$ for all $t \in[0, T]$. Since the estimates (2.35) hold for every $i=1, \ldots, n$, we conclude

$$
x_{i-1}\left(t, b_{i-1}\right) \leq z\left(t, b_{i-1}\right)<z\left(t, a_{i}\right) \leq x_{i}\left(t, a_{i}\right),
$$

proving that the funnels $\Gamma_{1}, \ldots, \Gamma_{n}$ remain disjoint.

We can now define a feedback control $u_{n}$ by setting

$$
u_{n}(t, x)=x_{i, t}(t, y) \quad \text { if }(t, x) \in \Gamma_{i} \text { for some } i \in\{1 \ldots, n\},
$$

and extending $u_{n}$ in a smooth way to the entire domain $[0, T] \times \mathbb{R}$.

Proposition 1. The above construction yields

$$
\lim _{n \rightarrow \infty} J\left(\mu_{n}, u_{n}\right)=J^{\operatorname{relax}}(\mu) .
$$

Therefore, for this example one has $J^{s}(\mu)=J^{\text {relax }}(\mu)$.

Proof. Writing the Euler-Lagrange equations for (2.3) and observing that the infimum cost for (2.4) is zero, we compute

$$
J^{\text {relax }}(\mu)=\int_{0}^{1} \int_{0}^{T}\left(z^{2}(t, y)+\dot{z}_{t}^{2}(t, y)\right) d t d y
$$

where $z$ is the function in (2.30). On the other hand,

$$
J\left(\mu_{n}, u_{n}\right)=\frac{n}{n-1} \sum_{i=1}^{n} \int_{a_{i}}^{b_{i}} \int_{0}^{T}\left(x_{i}^{2}(t, y)+x_{i, t}^{2}(t, y)+x_{i, y}^{2}(t, y)\right) d t d y,
$$

where, for $y \in\left[a_{i}, b_{i}\right]$, the quantity $x_{i}(t, y)$ is given by $(2.28)$. We observe that each function $x_{i}$ provides the global minimizer to the variational problem

$$
\text { minimize: } \quad J_{i}(w) \doteq \int_{a_{i}}^{b_{i}} \int_{0}^{T}\left(w^{2}(t, y)+w_{t}^{2}(t, y)+w_{y}^{2}(t, y)\right) d t d y
$$

among all functions $w \in W^{1,2}\left([0, T] \times\left[a_{i}, b_{i}\right]\right)$ such that

$$
w(0, y)=y \quad \text { for all } y \in\left[a_{i}, b_{i}\right]
$$

Consider the functions $w_{i}$ defined as follows. For $y \in\left[a_{i}, b_{i}\right]$, let

$$
w_{i}(t, y) \doteq \begin{cases}n t z\left(\frac{1}{n}, \frac{a_{i}+b_{i}}{2}\right)+(1-n t) y, & \text { if } t \in\left[0, n^{-1}\right], \\ z\left(t, \frac{a_{i}+b_{i}}{2}\right), & \text { if } t \in\left[n^{-1}, T\right] .\end{cases}
$$

For every $n \geq 1$ and $i \in\{1, \ldots, n\}$, it is easy to check that these functions satisfy the uniform bounds

$$
w_{i}(t, y) \in[0,1], \quad w_{i, y}(t, y) \in[0,1], \quad\left|w_{i, t}(t, y)\right| \leq M,
$$

for some uniform constant $M$. Hence the following estimates hold:

$$
\begin{gathered}
\int_{a_{i}}^{b_{i}} \int_{0}^{T} w_{i, y}^{2}(t, y) d t d y \leq \int_{a_{i}}^{b_{i}} \int_{0}^{1 / n} d t d y=\frac{b_{i}-a_{i}}{n} \\
\int_{a_{i}}^{b_{i}} \int_{0}^{1 / n}\left(w_{i}^{2}(t, y)+w_{i, t}^{2}(t, y)\right) d t d y \leq \frac{\left(1+M^{2}\right)\left(b_{i}-a_{i}\right)}{n} .
\end{gathered}
$$


Using the above inequalities and recalling that $\sum_{i=1}^{n}\left(b_{i}-a_{i}\right)=(n-1) / n$, since each $x_{i}:[0, T] \times\left[a_{i}, b_{i}\right] \mapsto \mathbb{R}$ provides a global minimizer, we obtain

$$
\begin{aligned}
J\left(\mu_{n}, u_{n}\right) \leq & \frac{n}{n-1} \sum_{i=1}^{n} \int_{a_{i}}^{b_{i}} \int_{0}^{T}\left(w_{i}^{2}(t, y)+w_{i, t}^{2}(t, y)+w_{i, y}^{2}(t, y)\right) d t d y \\
\leq & \frac{n}{n-1} \sum_{i=1}^{n} \frac{\left(2+M^{2}\right)\left(b_{i}-a_{i}\right)}{n} \\
& +\frac{n}{n-1} \sum_{i=1}^{n}\left(b_{i}-a_{i}\right) \int_{1 / n}^{T}\left(z^{2}\left(t, \frac{a_{i}+b_{i}}{2}\right)+z_{t}^{2}\left(t, \frac{a_{i}+b_{i}}{2}\right)\right) d t \\
\leq & \frac{\left(2+M^{2}\right)}{n}+\sum_{i=1}^{n}\left(a_{i+1}-a_{i}\right) \int_{0}^{T}\left(z^{2}\left(t, \frac{a_{i}+b_{i}}{2}\right)\right. \\
& \left.+z_{t}^{2}\left(t, \frac{a_{i}+b_{i}}{2}\right)\right) d t \doteq A_{n}+B_{n} .
\end{aligned}
$$

Letting $n \rightarrow \infty$, we clearly have $A_{n} \rightarrow 0$. On the other hand, $B_{n}$ is an approximate Riemann sum for the integral (2.38). Hence $\lim _{n \rightarrow \infty} B_{n}=J^{\text {relax }}(\mu)$. From (2.46) it follows

$$
\liminf _{n \rightarrow \infty} J\left(\mu_{n}, u_{n}\right) \leq J^{\text {relax }}(\mu),
$$

The converse inequality is clear.

Remark 2. In this example, the presence of gaps in the probability distributions $\mu_{n}$ is essential. Indeed, if we used the feedback controls $u_{n}$ in connection with the original probability $\mu$, uniformly distributed on $[0,1]$, the cost $J\left(\mu, u_{n}\right)$ would be very large. This is because, for initial data $b_{i}<x(0)<a_{i+1}$, along the trajectory $t \mapsto x(t)$ one can have $u_{x}(t, x(t))>>1$. This forces

$$
\xi(t)=\exp \left(\int_{0}^{t} u_{x}(s, x(s)) d s\right)
$$

to be very large, producing a large cost in (2.1). Although the probability of the initial data falling outside the intervals $\cup_{1 \leq i \leq n}\left[a_{i}, b_{i}\right]$ is very small, these few initial data determine a big increase in the expected cost in (2.1).

This example illustrates a case where

$$
J^{\text {relax }}(\mu)=J^{s}(\mu) \text {, but } J^{s}(\mu) \neq J(\mu) .
$$

The problems (2.3) and (2.8)-(2.9) both have regular solutions, but the minimization problem (1.14) does not. A minimizing sequence $u_{n}$ should have the form

$$
u_{n}(t, x)=u^{*}(t, x)+\tilde{u}_{n}(t, x)
$$

where $u^{*}$ is the optimal linear feedback in $(2.5)$, while $\left\|\tilde{u}_{n}\right\|_{\mathcal{C}^{0}} \rightarrow 0,\left\|\tilde{u}_{n}\right\|_{\mathcal{C}^{1}}=$ $\mathcal{O}(1)$

Remark 3. This first example suggests a general strategy for proving the equivalence $J^{\text {relax }}(\mu)=J^{s}(\mu)$. Namely: 
(i) Let $u_{\varepsilon}, v_{\varepsilon}$ be $\mathcal{C}^{2}$ feedbacks which achieve an almost optimal cost, in connection with the relaxed problem (1.11), (1.15). In other words, assume $\mathcal{J}\left(\mu ; u_{\varepsilon}, v_{\varepsilon}\right) \leq J^{\text {relax }}+\varepsilon$.

(ii) Split the support of the initial distribution $\mu$ into several small, disjoint intervals $\left[a_{i}, b_{i}\right]$, separated by small gaps. For each $i$, let $t \mapsto x_{i}(t)$ be the solution of $(1.15)$ with initial data $x_{i}(0)=\left(a_{i}+b_{i}\right) / 2$.

(iii) Define the linear feedback control

$$
u_{\varepsilon}^{i}(t, x) \doteq u_{\varepsilon}\left(t, x_{i}(t)\right)+v_{\varepsilon}\left(t, x_{i}(t)\right) \cdot\left(x-x_{i}(t)\right),
$$

and let $\mathcal{F}_{i}$ be the set of all solutions to the ODE

$$
\left\{\begin{array}{l}
\left.\dot{x}=f\left(t, x_{i}(t), \xi(t), u_{\varepsilon}^{i}(t, x)\right)\right), \\
\left.\dot{\xi}=g\left(t, x(t), \xi(t), u_{\varepsilon}^{i}(t, x)\right), v_{\varepsilon}\left(t, x_{i}(t)\right)\right),
\end{array}\right.
$$

with initial data $x(0) \in\left[a_{i}, b_{i}\right]$.

(iv) If the funnels

$$
\Gamma_{i} \doteq\left\{(t, x(t)) ; t \in[0, T], x(\cdot) \in \mathcal{F}_{i}\right\}, \quad i=1, \ldots, n,
$$

do not overlap, then one can define a new feedback by setting

$$
\tilde{u}(t, x) \doteq u_{\varepsilon}^{i}(t, x) \quad \text { if } \quad(t, x) \in \Gamma_{i},
$$

and extending $\tilde{u}$ in a smooth way on $\mathbb{R}^{2} \backslash \cup_{i} \Gamma_{i}$. By choosing the intervals $\left[a_{i}, b_{i}\right]$ sufficiently small, the cost provided by this feedback control $u^{*}$ can be rendered arbitrarily close to $J\left(\mu, u_{\varepsilon}, v_{\varepsilon}\right)$.

Here the fact that the funnels $\Gamma_{i}$ remain disjoint is essential. In the next section we look at a case where this property fails, and the two infimum costs $J^{\text {relax }}$ and $J^{s}$ do not coincide.

\section{A case of expanding funnels}

Example 2. We now consider the problem of minimizing the same quadratic functional as in (2.1), but subject to the dynamics

$$
\left\{\begin{array}{l}
\dot{x}=u, \\
\dot{\xi}=-\xi u_{x} .
\end{array}\right.
$$

Because of the negative sign in the second equation, we now have $\phi(t, x)=$ $\xi(t, x)$ for all $t, x$. Using again the variable $y$ to label characteristics, consider the problem (2.8), for a system with dynamics

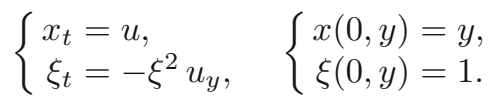

The evolution of the dual variables is determined by

$$
\left\{\begin{array} { l } 
{ \lambda _ { 1 , t } = - 2 x , } \\
{ \lambda _ { 2 , t } = 2 \xi \lambda _ { 2 } u _ { y } - 2 \xi , }
\end{array} \quad \left\{\begin{array}{l}
\lambda_{1}(T, y)=0, \\
\lambda_{2}(T, y)=0 .
\end{array}\right.\right.
$$


Therefore

$$
\begin{aligned}
\lambda_{1}(t, y)= & \int_{t}^{T} 2 x(\tau, y) d \tau, \quad \lambda_{2}(t, y)=\int_{t}^{T} \exp \\
& \times\left(-\int_{t}^{\tau} 2 \xi(s, y) u_{y}(s, y) d s\right) 2 \xi(\tau, y) d y
\end{aligned}
$$

The maximality condition takes the form

$$
u(t, \cdot)=\underset{\omega(\cdot)}{\operatorname{argmin}} \int_{0}^{1}\left[\lambda_{1}(t, y) \omega(y)-\lambda_{2}(t, y) \xi^{2}(t, y) \omega_{y}(y)+\omega^{2}(y)\right] d y .
$$

Assume that, for a fixed time $t$, the function $u=u(t, y)$ provides the minimum in (2.12). Then, for every smooth function $\varphi:[0,1] \mapsto \mathbb{R}$, setting $u^{(\varepsilon)}(y)=u(t, y)+\varepsilon \varphi(y)$ one should have

$$
\begin{aligned}
0= & \left.\frac{d}{d \varepsilon} \int_{0}^{1}\left[\lambda_{1}(t, y) u^{(\varepsilon)}(y)-\lambda_{2}(t, y) \xi^{2}(t, y) u_{y}^{(\varepsilon)}(y)+\left(u^{(\varepsilon)}\right)^{2}(y)\right] d y\right|_{\varepsilon=0} \\
= & \int_{0}^{1}\left[\lambda_{1}(t, y) \varphi(y)-\lambda_{2}(t, y) \xi^{2}(t, y) \varphi_{y}(y)+2 u(t, y) \varphi(y)\right] d y \\
= & \int_{0}^{1}\left[\lambda_{1}(t, y)+\left(\lambda_{2} \xi^{2}\right)_{y}(t, y)+2 u(t, y)\right] \varphi(y) d y+\lambda_{2}(t, 1) \xi^{2}(t, 1) \varphi(1) \\
& -\lambda_{2}(t, 0) \xi^{2}(t, 0) \varphi(0) .
\end{aligned}
$$

Since the function $\varphi$ can be arbitrary, this yields the Euler-Lagrange equations

$$
u(t, y)=-\frac{\lambda_{1}(t, y)+\left(\lambda_{2} \xi^{2}\right)_{y}(t, y)}{2} .
$$

together with the boundary conditions

$$
\left(\lambda_{2} \xi^{2}\right)(t, 0)=\left(\lambda_{2} \xi^{2}\right)(t, 1)=0 .
$$

Observe that (3.2) and (3.3) yield

$$
\left(\lambda_{2} \xi^{2}\right)_{t}=\left(2 \xi \lambda_{2} u_{y}-2 \xi\right) \xi^{2}-\lambda_{2} \xi^{2} u_{y}=-2 \xi^{3} .
$$

Differentiating both sides of (3.6) w.r.t. $t$ and using (3.8) one obtains

$$
u_{t}(t, y)=-\frac{\lambda_{1, t}(t, y)+\left(\lambda_{2} \xi^{2}\right)_{t, y}(t, y)}{2}=x+\left(\xi^{3}\right)_{y} .
$$

Using the identities

$$
x_{t t}(t, y)=u_{t}(t, y), \quad x_{y}(t, y)=\frac{1}{\phi(t, y)}=\frac{1}{\xi(t, y)},
$$

we thus recover the PDE

$$
x_{t t}+\frac{3 x_{y y}}{\left(x_{y}\right)^{4}}-x=0 .
$$

This is a nonlinear elliptic equation, to be solved on the rectangle $[0, T] \times$ $[0,1]$. From (3.2) and the terminal conditions in (3.3), using (3.6) one obtains the boundary conditions

$$
x(0, y)=y, \quad x_{t}(T, y)=u(T, y)=0 .
$$



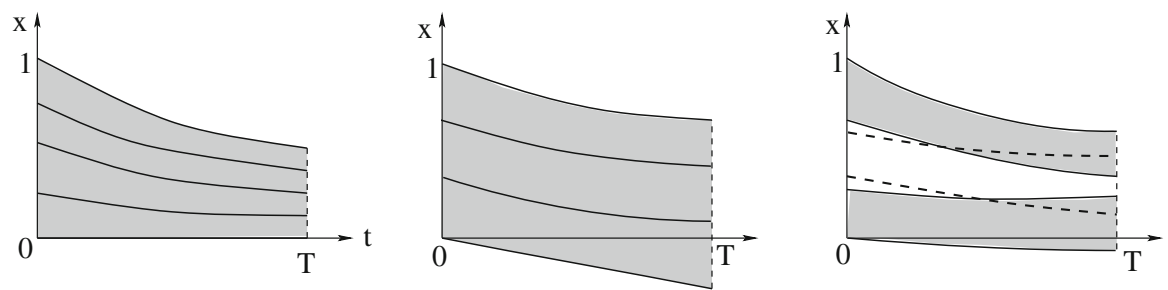

Figure 2. Left the optimal trajectories for the standard linear-quadratic optimization problem with dynamics (2.9) and cost (2.3) independent of $\xi$. Center for the system (3.1), the presence of a cost depending on $\xi$ renders more profitable a control where $u_{x}$ is positive. Hence the optimal solution should be supported on a larger interval. Right allowing gaps in the support of $\mu$ does not provide a way to achieve a lower cost, because in this case the funnels $\Gamma_{i}$ determined by nearoptimal feedback controls would overlap

Moreover,

$$
\left\{\begin{array}{l}
x_{y}(t, 0)=\frac{1}{\xi(t, 0)}=+\infty \\
x_{y}(t, 1)=\frac{1}{\xi(t, y)}=+\infty
\end{array}\right.
$$

because $0=\left(\lambda \xi^{2}\right)_{t}(t, 0)=-2 \xi^{3}(t, 0), 0=\left(\lambda \xi^{2}\right)_{t}(t, 1)=-2 \xi^{3}(t, 1)$ (Fig. 2).

In contrast with the optimal solution in Example 1, letting $T \rightarrow \infty$ the optimal trajectories do not converge to zero. Rather than $(2.24)$, we expect that the solution $x(t, y), \xi(t, y)$ will approach a steady state $(\bar{x}(y), \bar{\xi}(y))$. Because of the identity $\xi \cdot x_{y} \equiv 1$, the function $\bar{x}(\cdot)$ should provide a global minimizer to the variational problem

$$
\text { minimize: } \int_{0}^{1}\left[x^{2}(y)+\left(\frac{1}{x^{\prime}(y)}\right)^{2}\right] d y,
$$

the minimum being sought among all absolutely continuous, non-decreasing functions $x:[0,1] \mapsto \mathbb{R}$, with free boundary conditions. The Euler-Lagrange equations for this problem yield

$$
\begin{gathered}
x-\frac{3 x^{\prime \prime}}{\left(x^{\prime}\right)^{4}}=0, \\
\lim _{y \rightarrow 0+} x^{\prime}(y)=\lim _{y \rightarrow 1-} x^{\prime}(y)=+\infty .
\end{gathered}
$$

A solution to the above equations is found to be implicitly determined by

$$
\frac{x(y)}{2} \sqrt{\frac{2 \sqrt{3}}{\pi}-x^{2}(y)}+\frac{\sqrt{3}}{\pi} \arcsin \frac{\sqrt{\pi} x(y)}{\sqrt{2 \sqrt{3}}}=\sqrt{3}\left(y-\frac{1}{2}\right) .
$$


Observe that this function satisfies $x(y)=-x(1-y)$ for $y \in[0,1]$. As $t \rightarrow+\infty$, the measures $\mu^{(t)}$ approach a probability distribution $\mu^{\infty}$ which is symmetric w.r.t. the origin. Notice that, as $T \rightarrow+\infty$, in Example 1 the minimum cost remains bounded, while in Example 2 it approaches $+\infty$.

In this case, we could again consider the funnels $\Gamma_{i}$, defined as in (2.29), where now $x=x(t, y)$ is the solution of $(3.11)$ on $[0, T] \times\left[a_{i}, b_{i}\right]$, with boundary conditions

$$
\left\{\begin{array} { l } 
{ x ( 0 , y ) = y , } \\
{ x _ { t } ( T , y ) = 0 , }
\end{array} \quad \left\{\begin{array}{l}
x_{y}\left(a_{i}, t\right)=+\infty \\
x_{y}\left(b_{i}, t\right)=+\infty
\end{array}\right.\right.
$$

However, these funnels now overlap with each other (Fig. 2), and the definition (2.36) is not meaningful. For this example, we thus expect

$$
J(\mu)=J^{s}(\mu) \text {, but } J^{s}(\mu) \neq J^{\text {relax }}(\mu) .
$$

\section{A case where the width of the funnels can be controlled}

Example 3. We now consider a case where $f$ depends also on $\xi$, and one can use this additional variable in order to control the width of the funnels $\Gamma_{i}$, preventing their overlap. Consider again the optimization problem (2.1), but assume that the state of the system evolves according to

$$
\left\{\begin{array}{l}
\dot{x}=u+\xi \\
\dot{\xi}=u_{x}
\end{array}\right.
$$

with initial data

$$
x(0)=y, \quad \xi(0)=h(y) .
$$

As before, we assume that $y$ is a random variable uniformly distributed on the interval $[0,1]$. Otherwise stated, the probability measure $\mu$ has density $\phi=\chi_{[0,1]}$ w.r.t. Lebesgue measure. The corresponding relaxed system is

$$
\left\{\begin{array}{l}
\dot{x}=u+\xi \\
\dot{\xi}=v .
\end{array}\right.
$$

By (2.1) and (4.3), to achieve a global minimum one should have

$$
u=\xi=\frac{x_{t}}{2} .
$$

For each fixed $y$, writing the Euler-Lagrange equations we find that the optimal solution $t \mapsto x(t, y)$ to the relaxed problem solves the two-point boundary value problem

$$
\ddot{x}-2 x=0 \quad \text { for } t \in[0, T], \quad x(0, y)=y, \quad \dot{x}(T, y)=0 .
$$

The optimal solution is thus found to be

$$
\hat{x}(t, y)=\frac{e^{\sqrt{2}(t-2 T)}+e^{-\sqrt{2} t}}{1+e^{-2 \sqrt{2} T}} y .
$$


For this relaxed solution, the corresponding variables $u, \xi$ are given by

$$
\hat{u}(t, y)=\hat{\xi}(t, y)=\frac{\hat{x}_{t}(t, y)}{2}=\frac{e^{\sqrt{2}(t-2 T)}-e^{-\sqrt{2} t}}{1+e^{-2 \sqrt{2} T}} \frac{y}{\sqrt{2}} .
$$

On the other hand, for a fixed $y$, the map $\hat{v}(\cdot, y)$ should formally be given by the distributional derivative of the map $t \mapsto \hat{\xi}(t, y)$. This is a measure containing a point mass of size

$$
\hat{\xi}(0+, y)-h(y)=\frac{e^{-2 \sqrt{2} T}-1}{e^{-2 \sqrt{2} T}+1} \frac{y}{\sqrt{2}}-h(y)
$$

at the origin, while its restriction to the open set $\{t>0\}$ is absolutely continuous w.r.t. Lebesgue measure, with density

$$
\hat{v}(t, y)=\frac{1}{2} \hat{x}_{t t}(t, y)=\hat{x}(t, y)=\frac{e^{\sqrt{2}(t-2 T)}+e^{-\sqrt{2} t}}{1+e^{-2 \sqrt{2} T}} y .
$$

From the above analysis, we conclude that the infimum among all costs $J(\mu$, $u, v)$, with $u, v \in \mathcal{C}^{2}$, is provided by

$$
J^{\text {relax }}(\mu)=\int_{0}^{1} \int_{0}^{T}\left(\hat{x}^{2}(t, y)+\frac{1}{2} \hat{x}_{t}^{2}(t, y)\right) d t d y .
$$

A minimizing sequence of feedback controls $\left(u_{\nu}, v_{\nu}\right)_{\nu \geq 1}$ is provided by

$$
\begin{gathered}
u_{\nu}(t, x)=u(t, x)=\frac{e^{\sqrt{2}(t-2 T)}-e^{-\sqrt{2} t}}{e^{\sqrt{2}(t-2 T)}+e^{-\sqrt{2} t}} \frac{x}{\sqrt{2}}, \\
v_{\nu}(t, x)= \begin{cases}\nu \cdot\left(\frac{e^{-2 \sqrt{2} T}-1}{e^{-2 \sqrt{2} T}+1} \frac{x}{\sqrt{2}}-h(x)\right) & \text { if } t \in\left[0, \nu^{-1}\right], \\
x & \text { if } t \in\left[\nu^{-1}, T\right] .\end{cases}
\end{gathered}
$$

By performing a suitable cut-off, followed by a mollification, we achieve $u_{\nu}, v_{\nu} \in$ $\mathcal{C}^{2}$

This preliminary analysis shows that, for any $\varepsilon>0$, there exists smooth feedback controls $u^{*}=u^{*}(t, x)$ and $v^{*}=v^{*}(t, x)$ such that

$$
\mathcal{J}\left(\mu, u^{*}, v^{*}\right) \leq J^{\text {relax }}(\mu)+\varepsilon,
$$

and, calling $x=x^{*}(t, y), \xi=\xi^{*}(t, y)$ the corresponding solutions of (4.2)-(4.3), one has

$$
\begin{array}{cl}
\left\|x^{*}\right\|_{\mathcal{C}^{2}([0, T] \times[0,1])} \leq M_{0}, & \left\|\xi^{*}\right\|_{\mathcal{C}^{2}([0, T] \times[0,1])} \leq M_{0}, \\
\left\|u^{*}\right\|_{\mathcal{C}^{2}([0, T] \times \mathbb{R})} \leq M_{1}, & \left\|v^{*}\right\|_{\mathcal{C}^{2}([0, T] \times \mathbb{R})} \leq M_{1}, \\
x_{y}^{*}(t, y) \geq \rho_{0}>0, & \|h(y)\|_{\mathcal{C}^{2}([0,1])} \leq M_{2},
\end{array}
$$

for some constants $M_{0}, M_{1}, M_{2}, \rho_{0}$, possibly depending on $\varepsilon$. 
Proposition 2. In the above example one has $J^{s}(\mu)=J^{\text {relax }}(\mu)$.

Proof. Given $\varepsilon>0$, let $\left(u^{*}, v^{*}\right)$ be a pair of generalized feedback controls for which all the estimates $(4.10)-(4.12)$ hold. To prove Proposition 2 , we need to show that there exists a measure $\tilde{\mu}$ with density $\tilde{\phi}$ satisfying $\|\tilde{\phi}-\phi\|_{\mathbf{L}^{1}} \leq \varepsilon$ and a feedback control $\tilde{u} \in \mathcal{C}^{2}$ such that

$$
J(\tilde{u}, \tilde{\mu}) \leq \mathcal{J}\left(u^{*}, v^{*}, \mu\right)+\varepsilon .
$$

1. Consider the augmented system of ODEs

$$
\left\{\begin{array} { l } 
{ \dot { x } = u + \xi , } \\
{ \dot { \xi } = v , } \\
{ \dot { \eta } = \eta v + z , } \\
{ \dot { z } = w \eta , }
\end{array} \quad \left\{\begin{array}{l}
x(0, y)=y, \\
\xi(0, y)=h(y), \\
\eta(0, y)=1, \\
z(0, y)=h^{\prime}(y) .
\end{array}\right.\right.
$$

Here we think of $\eta=x_{y}$ and $z=\xi_{y}$ as an additional variable, while $v=u_{x}$, $w=v_{x}=u_{x x}$ are additional controls. Notice that the last two ODEs in (4.14) follow from

$$
\left(x_{y}\right)_{t}=u_{y}+\xi_{y}=u_{x} x_{y}+\xi_{y}, \quad\left(\xi_{y}\right)_{t}=v_{y}=v_{x} x_{y} .
$$

2. For $n$ large, consider the probability distribution $\mu_{n}$ having density

$$
\phi_{n}(x) \doteq\left\{\begin{array}{cc}
\frac{n}{n-1} & \text { if } x \in\left[\frac{i-1}{n}, \frac{i}{n}-\frac{1}{n^{2}}\right] \doteq\left[a_{i}, b_{i}\right] \\
& \text { for some } i \in\{1, \ldots, n\} \\
0 & \text { otherwise. }
\end{array}\right.
$$

For $i=1, \ldots, n$, denote by $t \mapsto x_{i}(t) \doteq x^{*}\left(t, a_{i}\right), t \mapsto \xi_{i}(t) \doteq \xi^{*}\left(t, a_{i}\right)$ the components of the solution of (4.2)-(4.3) with $y=a_{i}$. As a first attempt, one may construct the feedback $\tilde{u}$ by setting

$$
\tilde{u}(t, x) \doteq u^{*}\left(t, x_{i}(t)\right)+v^{*}\left(t, x_{i}(t)\right) \cdot\left(x-x_{i}(t)\right)
$$

for $x \approx x_{i}(t)$. For $y \in\left[a_{i}, b_{i}\right]$, call $t \mapsto(\tilde{x}(t, y), \tilde{\xi}(t, y))$ the solution of (4.1)-(4.2), with $u=\tilde{u}$ given by (4.16). Observe that this construction yields

$$
\begin{aligned}
& \tilde{x}\left(t, a_{i}\right)=x_{i}(t) \doteq x^{*}\left(t, a_{i}\right), \quad \tilde{\xi}\left(t, a_{i}\right)=\xi_{i}(t) \doteq \xi^{*}\left(t, a_{i}\right), \\
& t \in[0, T], 1 \leq i \leq n .
\end{aligned}
$$

Introducing the tubes

$$
\Gamma_{i} \doteq\left\{(t, \tilde{x}(t, y)) ; t \in[0, T], y \in\left[a_{i}, b_{i}\right]\right\}
$$

one may hope to define $\tilde{u}(t, x)$ by (4.16) for $(t, x) \in \Gamma_{i}$, and extend $\tilde{u}$ in a smooth way on the complementary set $([0, T] \times \mathbb{R}) \backslash \cup_{i} \Gamma_{i}$. Notice that (4.12) implies $x_{1}(t)<x_{2}(t)<\cdots<x_{n}(t)$, so that the centers of these tubes do not cross each other. Unfortunately, in the present situation there is no guarantee that the tubes $\Gamma_{i}$ remain disjoint for all $t \in[0, T]$. We thus need to refine our construction, relying on a global controllability property of the system (4.14). 


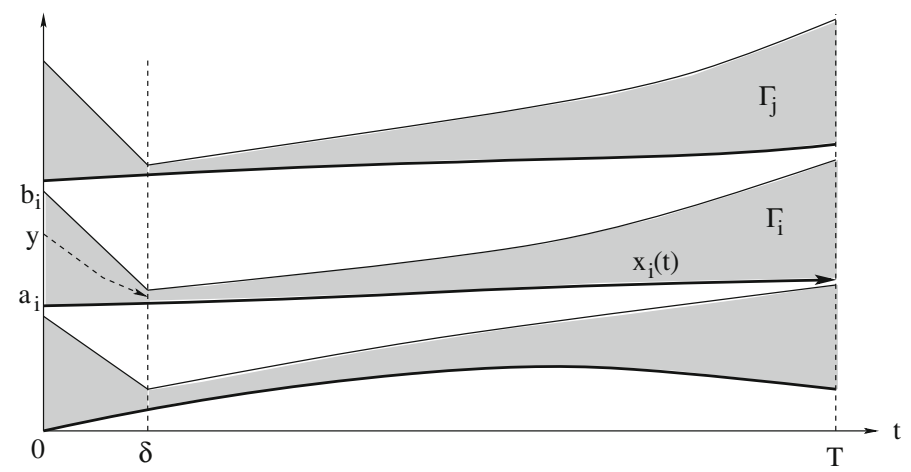

Figure 3. On the initial time interval $[0, \delta]$ a feedback control is implemented such that all initial points $y \in\left[a_{i}, b_{i}\right]$ are steered inside a very small neighborhood of the point $x_{i}(\delta)=x\left(\delta, a_{i}\right)$. Since at time $t=\delta$ we have $x_{y} \approx 0$ and $\xi_{y} \approx 0$ inside each tube $\Gamma_{i}$, this guarantees that for $t \in[\delta, T]$ the tubes $\Gamma_{i}$ remain mutually disjoint

On a small time interval $[0, \delta]$, we will construct a feedback $\tilde{u}$ such that the corresponding solution of (4.1)-(4.2) satisfies

$$
\left|\tilde{x}(\delta, y)-x_{i}(\delta)\right|<\epsilon, \quad\left|\tilde{\xi}(\delta, y)-\xi_{i}(\delta)\right|<\epsilon, \quad \text { for all } y \in\left[a_{i}, b_{i}\right]
$$

If (4.18) holds, with $\epsilon<<n^{-1}$ suitably small, then for $t \in[\delta, T]$, and $x \approx$ $x_{i}(t)$ the definition (4.16) will provide a feedback with the desired properties. To achieve (4.18) we shall construct a feedback such that $\tilde{x}_{y}(\delta, y) \approx 0$ and $\tilde{\xi}_{y}(\delta, y) \approx 0$, for all $y \in\left[a_{i}, b_{i}\right]$ (Fig. 3$)$.

3. Let $\delta>0$ be given. Relying on the controllability of the ODE (4.14), for $0 \leq t \leq \delta$ and $i \in\{1, \ldots, n\}$, we construct control functions $u_{i}(\cdot), v_{i}(\cdot), w_{i}(\cdot)$ such that the solution of the Cauchy problem

$$
\left\{\begin{array} { l } 
{ \dot { x } = u _ { i } + \xi , } \\
{ \dot { \xi } = v _ { i } , } \\
{ \dot { \eta } = \eta v _ { i } + z , } \\
{ \dot { z } = \eta w _ { i } , }
\end{array} \quad \left\{\begin{array}{l}
x(0)=a_{i}, \\
\xi(0)=h\left(a_{i}\right), \\
\eta(0)=1, \\
z(0)=h^{\prime}\left(a_{i}\right) .
\end{array}\right.\right.
$$

satisfies $x(t)=x_{i}(t) \doteq x^{*}\left(t, a_{i}\right)$ for all $t \in[0, \delta]$ and moreover

$$
\xi(\delta)=\xi_{i}(\delta) \doteq \xi^{*}\left(\delta, a_{i}\right), \quad \eta(\delta) \approx 0, \quad z(\delta) \approx 0 .
$$

Solutions of (4.19) are more conveniently found using the variables

$$
X=\ln \eta, \quad Y=\frac{z}{\eta}
$$


which evolve according to

$$
\left\{\begin{array} { l } 
{ \dot { X } = \frac { \dot { \eta } } { \eta } = v _ { i } + Y , } \\
{ \dot { Y } = \frac { \dot { z } } { \eta } - \frac { z } { \eta } \frac { \dot { \eta } } { \eta } = w _ { i } - Y ^ { 2 } - Y v _ { i } \doteq \tilde { w } _ { i } , }
\end{array} \quad \left\{\begin{array}{l}
X(0)=0, \\
Y(0)=h^{\prime}\left(a_{i}\right) .
\end{array}\right.\right.
$$

We regard $\tilde{w}_{i}$ as an independent control function. Clearly, we can assign the controls $v_{i}(\cdot), \tilde{w}_{i}(\cdot)$ arbitrarily, then compute the solution of (4.21) and define the control $w_{i}(t) \doteq \tilde{w}_{i}(t)+Y^{2}(t)+Y(t) v_{i}(t)$.

To achieve (4.18), we use the controls

$$
\begin{gathered}
v_{i}(t) \doteq \begin{cases}\frac{-1+\xi_{i}(\delta)-\xi_{i}(0)}{\delta} & 0 \leq t<\frac{\delta}{2}, \\
\frac{1+\xi_{i}(\delta)-\xi_{i}(0)}{\delta} & \frac{\delta}{2} \leq t \leq \delta,\end{cases} \\
\tilde{w}_{i}(t) \doteq-\frac{1}{\delta^{3}}, \quad 0 \leq t \leq \delta,
\end{gathered}
$$

while the control $u_{i}$ is defined as

$$
u_{i}(t) \doteq u^{*}\left(t, x_{i}(t)\right)+\xi_{i}(t)-\left[\xi_{i}(0)+\int_{0}^{t} v_{i}(s) d s\right] \quad t \in[0, \delta] .
$$

The corresponding solution of (4.21) is

$$
\left\{\begin{array}{l}
X(t)=\int_{0}^{t} v_{i}(s) d s+\int_{0}^{t}\left(h^{\prime}\left(a_{i}\right)-\frac{s}{\delta^{3}}\right) d s \\
Y(t)=h^{\prime}\left(a_{i}\right)-\frac{t}{\delta^{3}}
\end{array}\right.
$$

In particular, at $t=\delta$ one has

$$
X(\delta)=\xi_{i}(\delta)-\xi_{i}(0)+h^{\prime}\left(a_{i}\right) \delta-\frac{1}{2 \delta}, \quad Y(\delta)=h^{\prime}\left(a_{i}\right)-\frac{1}{\delta^{2}}
$$

Going back to original variables $\eta, z$, one obtains

$$
\left\{\begin{array}{l}
\eta(\delta)=\exp (X(\delta))=\exp \left(\xi_{i}(\delta)-\xi_{i}(0)+h^{\prime}\left(a_{i}\right) \delta-\frac{1}{2 \delta}\right), \\
z(\delta)=Y(\delta) \eta(\delta)=\left(h^{\prime}\left(a_{i}\right)-\frac{1}{\delta^{2}}\right) \exp \left(\xi_{i}(\delta)-\xi_{i}(0)+h^{\prime}\left(a_{i}\right) \delta-\frac{1}{2 \delta}\right) .
\end{array}\right.
$$


Moreover, by the definition of $\tilde{w}$ in (4.21), the control $w_{i}=\tilde{w}_{i}+Y^{2}+Y v_{i}$ is given by

$$
w_{i}(t)=\left\{\begin{array}{l}
-\frac{1}{\delta^{3}}+\left(h^{\prime}\left(a_{i}\right)-\frac{t}{\delta^{3}}\right)^{2}+\left(\frac{-1+\xi_{i}(\delta)-\xi_{i}(0)}{\delta}\right)\left(h^{\prime}\left(a_{i}\right)-\frac{t}{\delta^{3}}\right), \\
0 \leq t<\frac{\delta}{2}, \\
-\frac{1}{\delta^{3}}+\left(h^{\prime}\left(a_{i}\right)-\frac{t}{\delta^{3}}\right)^{2}+\left(\frac{1+\xi_{i}(\delta)-\xi_{i}(0)}{\delta}\right)\left(h^{\prime}\left(a_{i}\right)-\frac{t}{\delta^{3}}\right), \\
\frac{\delta}{2} \leq t \leq \delta .
\end{array}\right.
$$

By (4.22), (4.23), and (4.25), for $\delta>0$ sufficiently small we have the estimates

$$
\left|v_{i}(t)\right| \leq \frac{2}{\delta}, \quad|Y(t)| \leq \frac{2}{\delta^{2}}, \quad\left|w_{i}(t)\right| \leq \frac{5}{\delta^{4}}, \quad \text { for all } t \in[0, \delta] .
$$

Moreover, by (4.24), the solution $(x(\cdot), \xi(\cdot))$ of $(4.19)$ satisfies

$$
\dot{x}(t)=u_{i}(t)+\xi(t)=u^{*}\left(t, x_{i}(t)\right)+\xi_{i}(t)=\dot{x}_{i}(t), \quad \text { for all } t \in[0, \delta] .
$$

4. On a suitable neighborhood of each trajectory $t \mapsto x_{i}(t)$, we then define the feedback control $\tilde{u}$ as

$$
\tilde{u}(t, x) \doteq \begin{cases}u_{i}(t)+\left(x-x_{i}(t)\right) \cdot v_{i}(t)+\frac{\left(x-x_{i}(t)\right)^{2}}{2} \cdot w_{i}(t), & t \in[0, \delta], \\ u^{*}\left(t, x_{i}(t)\right)+\left(x-x_{i}(t)\right) \cdot v^{*}\left(t, x_{i}(t)\right), & t \in[\delta, T] .\end{cases}
$$

The corresponding solution of (4.1) with initial data (4.2) will be denoted by $t \mapsto(\tilde{x}(t, y), \tilde{\xi}(t, y))$. We can then extend $\tilde{u}$ in a smooth way (w.r.t. the $x$ variable) on the complement of the set $\cup_{1 \leq i \leq n} \Gamma_{i}$. Notice that, by choosing $n=n(\delta) \gg \delta^{-1}$, we can achieve the convergence

$$
\left\|\tilde{u}-u^{*}\right\|_{\mathbf{L}^{\infty}([\delta, T] \times \mathbb{R})} \rightarrow 0 \quad \text { as } \delta \rightarrow 0 .
$$

For every $i \in\{1, \ldots, n\}$, the above construction yields

$$
\begin{gathered}
\tilde{x}\left(t, a_{i}\right)=x_{i}(t) \quad t \in[0, T] . \\
\left|\tilde{\xi}\left(t, a_{i}\right)\right|=\left|\xi_{i}(0)+\int_{0}^{t} v_{i}(s) d s\right| \leq M_{0}(1+\delta)+1 \quad t \in[0, \delta], \\
\tilde{\xi}\left(\delta, a_{i}\right)=\xi_{i}(\delta) .
\end{gathered}
$$

We claim that, by choosing $\delta>0$ sufficiently small one can achieve

$$
\begin{gathered}
0<\tilde{x}_{y}\left(\delta, a_{i}\right)<\delta, \quad\left|\tilde{\xi}_{y}\left(\delta, a_{i}\right)\right|<\delta, \\
0<\tilde{x}_{y}\left(t, a_{i}\right)<x_{y}^{*}\left(t, a_{i}\right) \quad \text { for all } t \in[0, \delta] .
\end{gathered}
$$

To prove (4.33) we observe that the functions $\eta(t)=\tilde{x}_{y}\left(t, a_{i}\right)$ and $z(t)=$ $\tilde{\xi}_{y}\left(t, a_{i}\right)$ satisfy the system of ODEs (4.19). Hence the bounds (4.33) are an immediate consequence of (4.27), because $e^{-1 / 2 \delta}<<\delta$ for $\delta>0$ small. 
Next, by (4.11) we have $\left\|x_{y t}^{*}\right\|_{\mathcal{C}^{0}} \leq\left\|x^{*}\right\|_{\mathcal{C}^{2}} \leq M_{0}$. Since $x_{y}^{*}\left(0, a_{i}\right)=1$, this implies

$$
x_{y}^{*}\left(t, a_{i}\right) \geq 1-M_{0} t
$$

For $t \in[0, \delta / 2]$ by (4.25) we have

$$
X(t)=\frac{-1+\xi_{i}(\delta)-\xi_{i}(0)}{\delta} t+h^{\prime}\left(a_{i}\right) t-\frac{t^{2}}{2 \delta^{3}} \leq-2 M_{0} t \leq \ln \left(x_{y}^{*}\left(t, a_{i}\right)\right),
$$

provided that $\delta>0$ is small enough. On the other hand, for $y \in[\delta / 2, \delta]$, we obtain

$$
\begin{aligned}
X(t) & =\int_{0}^{\delta / 2} v_{i}(s) d s+\int_{\delta / 2}^{t} v_{i}(s) d s+\int_{0}^{t}\left(h^{\prime}\left(a_{i}\right)-\frac{s}{\delta^{3}}\right) \\
& \leq 1+\left|\xi_{i}(\delta)-\xi_{i}(0)\right|+M_{2} \delta-\frac{1}{8 \delta} \leq-2 M_{0} t .
\end{aligned}
$$

Therefore, always assuming that $\delta>0$ is sufficiently small, for all $t \in[0, \delta]$ we have

$$
0 \leq \tilde{x}_{y}\left(t, a_{i}\right)=\exp (X(t)) \leq x_{y}^{*}\left(t, a_{i}\right)
$$

An entirely similar estimate can be proved for every initial point $y \in\left[a_{i}, b_{i}\right]$. Provided that $\delta>0$ is sufficiently small, we thus conclude

$$
\begin{aligned}
& 0<\tilde{x}_{y}(\delta, y)<\delta, \quad\left|\tilde{\xi}_{y}(\delta, y)\right|<\delta, \quad \text { for all } y \in\left[a_{i}, b_{i}\right], \\
& \tilde{x}_{y}(t, y) \leq x_{y}^{*}(t, y) \quad \text { for all } t \in[0, \delta], y \in\left[a_{i}, b_{i}\right] .
\end{aligned}
$$

5. The estimate (4.38) shows that on the initial time interval $[0, \delta]$ the tubes $\Gamma_{i}$ defined as in (4.17) do not overlap with each other. Next, we check that the tubes $\Gamma_{i}$ remain disjoint also for $t \in[\delta, T]$.

The first inequality in (4.12) implies

$$
x_{i+1}(t)-x_{i}(t) \geq \frac{\rho_{0}}{n} \quad \text { for all } i \in\{0, \ldots, n-1\} .
$$

Observe that, by (4.1) and (4.30),

$$
\frac{\partial}{\partial t}\left(\left|\tilde{x}_{y}(t, y)\right|+\left|\tilde{\xi}_{y}(t, y)\right|\right) \leq\left(v^{*}\left(t, x_{i}(t)\right)+1\right) \cdot\left(\left|\tilde{x}_{y}(t, y)\right|+\left|\tilde{\xi}_{y}(t, y)\right|\right) .
$$

Therefore the bound (4.11) on $v^{*}$ together with (4.33) yields

$$
\tilde{x}_{y}(t, y) \leq e^{\left(M_{1}+1\right) T}\left(\left|\tilde{x}_{y}(\delta, y)\right|+\left|\tilde{\xi}_{y}(\delta, y)\right|\right) \quad \text { for all } t \in[\delta, T]
$$

For $y \in\left[a_{i}, b_{i}\right]$ and $t \in[\delta, T]$ we have

$$
\tilde{x}(t, y)-x_{i}(t) \leq \int_{a_{i}}^{y} \tilde{x}_{y}(t, z) d \tilde{z} \leq\left(b_{i}-a_{i}\right) \cdot \sup _{y \in[0,1]} \tilde{x}_{y}(t, y) \leq \frac{\rho_{0}}{n},
$$

provided that

$$
e^{\left(M_{1}+1\right) T} \cdot \sup _{y}\left(\left|\tilde{x}_{y}(\delta, y)\right|+|\tilde{\xi}(\delta, y)|\right) \leq \rho_{0} .
$$


Recalling (4.33) we can now choose $\delta>0$ small enough so that $e^{\left(M_{1}+1\right) T} 4 \delta<$ $\rho_{0}$. Then we choose $n=n(\delta) \gg 1 / \delta$ large enough so that, by continuity, the estimates

$$
\left(\left|\tilde{x}_{y}(\delta, y)\right|+|\tilde{\xi}(\delta, y)|\right)<4 \delta<\frac{\rho_{0}}{e^{\left(M_{1}+1\right) T}}
$$

remain valid for every $y \in\left[a_{i}, b_{i}\right], i=1, \ldots, n$. By (4.39) and (4.41), this implies that the tubes $\Gamma_{i}$ remain mutually disjoint also for $t \in[\delta, T]$.

6. It is clear that the sequence of densities $\phi_{n}$ in (4.15) converges to $\phi=\chi_{[0,1]}$ as $n \rightarrow \infty$. Having chosen $\delta>0$ and $n=n(\delta) \gg \delta^{-1}$ as before, let $\tilde{u}=\tilde{u}(t, x)$ be a feedback control satisfying (4.30) on each tube $\Gamma_{i}$, extended in a smooth way outside the union $\cup_{i=1}^{n} \Gamma_{i}$. It remains to show that, as $\delta \rightarrow 0$, the expected cost for the feedback $\tilde{u}$ approaches $\mathcal{J}\left(u^{*}, v^{*}, \mu\right)$. Indeed, on the initial interval $[0, \delta]$, by $(4.32)$ all functions $\tilde{x}, \tilde{\xi}, \tilde{u}$ remain uniformly bounded as $\delta \rightarrow 0$. Therefore

$$
\frac{n}{n-1} \cdot \sum_{i=1}^{n} \int_{0}^{\delta} \int_{a_{i}}^{b_{i}}\left(\tilde{x}^{2}(t, y)+\tilde{\xi}^{2}(t, y)+\tilde{u}^{2}(t, \tilde{x}(t, y))\right) d y d t \leq C \delta
$$

To see what happens on the remaining interval $[\delta, T]$, consider the quantities

$$
\begin{aligned}
& A_{n} \doteq \frac{n}{n-1} \cdot \sum_{i=1}^{n} \int_{\delta}^{T} \int_{a_{i}}^{b_{i}}\left(\tilde{x}^{2}(t, y)+\tilde{\xi}^{2}(t, y)+\tilde{u}^{2}(t, \tilde{x}(t, y))\right) d y d t \\
& B_{n} \doteq \frac{1}{n} \cdot \sum_{i=1}^{n} \int_{\delta}^{T}\left(\left(x^{*}\right)^{2}\left(t, a_{i}\right)+\left(\xi^{*}\right)^{2}\left(t, a_{i}\right)+\left(u^{*}\right)^{2}\left(t, x_{i}(t)\right)\right) d y d t .
\end{aligned}
$$

Recalling (4.31) we have $\left|A_{n}-B_{n}\right| \rightarrow 0$ as $\delta \rightarrow 0$ and $n=n(\delta) \rightarrow \infty$. Moreover,

$$
\begin{aligned}
\lim _{n \rightarrow \infty} B_{n} & =\int_{0}^{T} \int_{0}^{1}\left(\left(x^{*}\right)^{2}(t, y)+\left(\xi^{*}\right)^{2}(t, y)+\left(u^{*}\right)^{2}\left(t, x^{*}(t, y)\right)\right) d y d t \\
& =\mathcal{J}\left(u^{*}, v^{*}, \mu\right) .
\end{aligned}
$$

This completes the proof.

Remark 4. We point out a fundamental difference between the first two examples and this last one. Namely, consider the system of four ODEs, obtained by adding to (1.15) two additional equations for the variables $\alpha(t)=x_{y}(t, y)$ and $\beta(t)=\xi_{y}(t, x)$.

$$
\left\{\begin{array}{l}
\dot{x}=f, \\
\dot{\xi}=g, \\
\dot{\alpha}=\left(f_{x}+f_{u} v\right) \alpha+f_{\xi} \beta, \\
\dot{\beta}=\left(g_{x}+g_{u} v+g_{u_{x}} w\right) \alpha+g_{\xi} \beta .
\end{array}\right.
$$

Here $v=u_{x}$ and $w=u_{x x}$ are regarded as independent control functions. In the first two examples this system is not controllable. Indeed, no matter what controls are implemented, in Example 1 we always have $\xi(t)-\alpha(t) \equiv 0$, while in Example 2 one has $\xi(t) \cdot \alpha(t) \equiv 1$. On the other hand, in Example 3 there 
is no functional relation between $\xi$ and $\alpha$. We expect that this controllability property of the extended system (4.43) should play a key role, determining the equality between the minimal $\operatorname{costs} J^{s}(\mu)$ and $J^{\text {relax }}(\mu)$.

\section{References}

[1] Basar, T., Olsder, G.J.: Dynamic Noncooperative Game Theory. Reprint of the 2nd edn. SIAM, Philadelphia (1999)

[2] Bressan, A., Wei, D.: Non classical problems of optimal feedback control. J. Differ. Equ. 253, 1111-1142 (2012)

[3] Cesari, L.: Optimization: Theory and Applications. Springer, Berlin (1983)

[4] Dockner, E.J., Jorgensen, S., Long, N.V., Sorger, G.: Differential Games in Economics and Management Science. Cambridge University Press, Cambridge (2000)

[5] Jungers, M., Trélat, E., Abou-Kandil, H.: Min-max and min-min Stackelberg strategies with closed-loop information structure. J. Dynam. Control Syst. 17, 387-425 (2011)

[6] Medanic, J.: Closed-loop Stackelberg strategies in linear-quadratic problems. In: IEEE Trans. Autom. Control 23, 632-637 (1978)

[7] Miller, B.M., Rubinovich, Y.E.: Impulsive Control in Continuous and DiscreteContinuous Systems. Kluwer, New York (2003)

[8] Papavassilopoulos, G.P., Cruz, J.B.: Nonclassical control problems and Stackelberg games. IEEE Trans. Autom. Control 24, 155-166 (1979)

[9] Protter, M.H., Weinberger, H.F.: Maximum Principles in Differential Equations. Prentice Hall (1967)

[10] Rishel, R.W.: An extended Pontryagin principle for control systems whose control laws contain measures. SIAM J. Control 3, 191-205 (1965)

[11] Silva, G.N., Vinter, R.B.: Measure driven differential inclusions. J. Math. Anal. Appl. 202, 727-746 (1996)

Alberto Bressan and Deling Wei

Department of Mathematics

Penn State University

University Park

PA 16802

USA

e-mail: bressan@math.psu.edu

Deling Wei

e-mail: wei@math.psu.edu

Received: 10 December 2011.

Accepted: 14 March 2012. 